\title{
Helium Diffraction as a Probe of Structure and Proton Order on Model Ice-Surfaces
}

\author{
N. Avidor* and W. Allison \\ Cavendish Laboratory, University of Cambridge, JJ Thomson Ave, Cambridge, CB30HE, \\ United Kingdom \\ E-mail: na364@cam.ac.uk
}

\begin{abstract}
Helium diffraction has the potential to reveal the degree of proton order at an ice surface, and has been used in the past to benchmark theoretical work. We demonstrate that previous calculations do not represent the diffraction experiment to a sufficient degree of accuracy. By combining a realistic helium-water potential with quantum calculations using exact close-coupling methods we demonstrate that the scattering is strongly energy dependent. Proton order may be inferred best from selective adsorption resonances of the helium atom, which involve multiple scattering. We use the results to discuss the validity of the latest assumptions for the ice Ih surface with respect to proton ordering.
\end{abstract}

\section{Graphical TOC Entry}

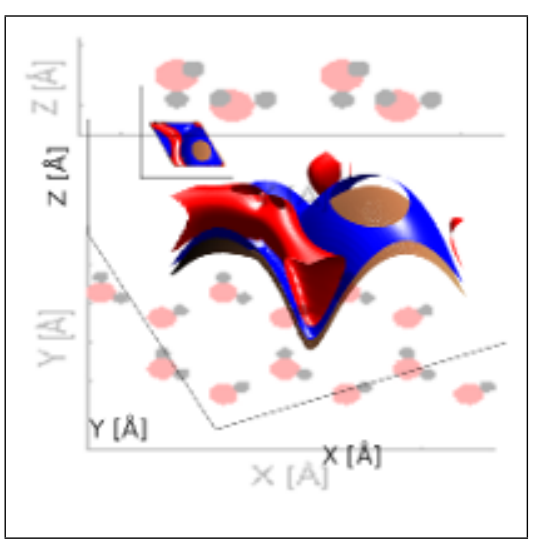

\section{Keywords}

American Chemical Society, Helium Atom Scattering, Water, Ice, Surface Science, CloseCoupled Equations, Proton Order, Eikonal 
The molecular properties of water at surfaces are a fundamentally important topic in surface science. ${ }^{1-3}$ A central challenge, which is of relevance in many fields, ${ }^{4}$ is the degree to which the water-protons are ordered at the surface of ice.

The structure of ice Ih, the stable crystalline form of ice under ambient conditions, is fourcoordinated with oxygen atoms adopting the lattice points. The structure can be viewed as hexagonal double-layered networks ("bilayers"), bonded to each-other perpendicular to the (0001) direction. At temperatures above $72 \mathrm{~K}$, the dipoles of the water molecules remain disordered, while at cryogenic temperatures it may form an ordered structure known as ice IX. ${ }^{5}$ The common picture at the surface however, is that the water molecules maintain protonorder even near the pre-melting temperature. Recent theoretical works suggest that either a 'Fletcher' striped model dominates the surface, ${ }^{6,7}$ or that a Fletcher striped model is one of the favoured structures. ${ }^{8-10}$

From the experimental point of view, the challenge in studying proton-order at water ad-layers arises not only from the complexity of the structures themselves but also because of fundamental limitations in the experimental probes we have available. Imaging techniques like STM can be used for studying ice only at low temperatures were the studied surface is typically of ice-IX, rather than ice-Ih. Furthermore, in thick ice films, interactions between the surface and the tip become more important. 1,2,11,12 Electron scattering methods such as LEED are mostly insensitive to the hydrogen orientation. ${ }^{13-16}$ However, in some cases, LEED measurements may be used as a reference for hydrogen-sensitive techniques like Helium Atom Scattering (HAS), where a difference in the symmetry measured with the two techniques may reveal the orientation of the water dipoles. ${ }^{16,17}$

Recent theoretical studies of the ice surface $^{6,8-10}$ have benchmarked their data against existing Helium Atom scattering ${ }^{18-20}$ (HAS), and Sum Frequency Generation ${ }^{21,22}$ (SFG) measurements. In conducting SFG experiments, special attention is taken to avoid local and temporal melting of the surface due to the high energies used. However, HAS is known to be a gentle probe of the surface, with low beam energies in the order of a few $\mathrm{meV}$. On the other-hand, based on approximated scattering simulations, it has been argued that HAS is insensitive to the proton order. ${ }^{19}$ In the present work we demonstrate that the assumptions underlying previous arguments are too simplistic.

To avoid computationally-expensive exact scattering simulations, approximations are usually made. The most common approach is to assume a Hard-Corrugated Wall (HCW) potential, an adequate assumption in cases where the corrugation of the potential is weak $(<10 \%)$, and the attractive part is negligible (usually for beam energies much higher than the He-surface potential well). A further common approximation, is to neglect multiple scattering and use the eikonal formula. ${ }^{23}$ In cases were the interaction potential is highly corrugated and the attractive well is important, exact solution to the Schrödinger equation for realistic 3D potentials is necessary in order to simulate the scattering event. ${ }^{24}$

Exact scattering calculations ${ }^{24}$ for molecular adsorbates are rare and to the best of our knowledge were used only in the study of CO adsorbed on $\mathrm{Cu}(100),{ }^{25}$ and $\mathrm{Pt}(111) .{ }^{26}$ In both studies, the authors used a He-CO gas-phase potential to construct a "pair-potential". Riley et. al. ${ }^{25}$ showed that even without tuning the summed pair-potential, a good description of the experiment is achieved, including boundstate resonances. They also showed that minor tuning to the average (DC) component of the potential provides a more precise description of the experiment.

Here, we explore the benefit from quantitative analysis of HAS measurements of water at surfaces. We construct a He-surface interaction potential out of He-water gas-phase potentials, and study the effect of structure on the scattering pattern. We focus on proton ordering. The results shows that diffraction is highly depended on the beam energy, and exhibits strong bound state resonance effects that depend on proton-order. We discuss the relevance of this work to the evolving picture of the surface of 
ice. ${ }^{6,8-10,18,19}$

The Helium-surface interaction potentials described in this work were calculated by summing-up individual He-water gas-phase potentials for each water molecule in the unit cell ("pair potential"). Some $120 \times 120$ neighbour unit-cells were included in calculating the potential. Using a gas-phase potential for the He-water interaction is justified as intact water molecules at surfaces are only slightly distorted with respect to the gas-phase. ${ }^{2}$ The specific potential we used as the building block for the He-surface interaction was calculated by Hodges et al., ${ }^{27}$ which was used successfully to explain several gas-phase experiments. The summation of pair-potentials is restricted to the outermost layer where most structural information is contained. Any contribution from deeper layers will add an average offset to the potential with little structural contribution that is not considered in the present work.

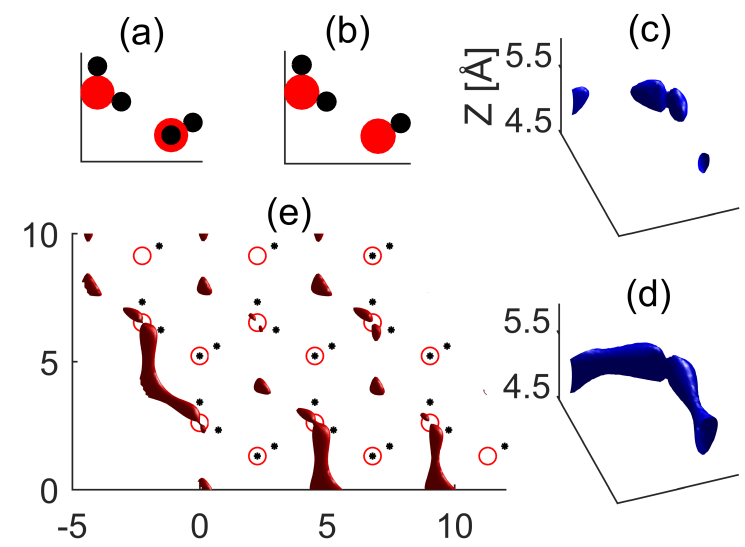

Figure 1: (a)\&(b) H-up and H-down ice bilayers. Large red (small black) circles represent oxygen (hydrogen) atoms. (c,d) Isosurfaces of $\mathrm{V}=-10 \mathrm{meV}$ for the $\mathrm{H}$-up and $\mathrm{H}$ down models respectively. (e) Iso-surface of $\mathrm{V}=-10 \mathrm{meV}$ superimposed on-top of a protondisordered super-cell with a ratio of $6 / 3 \mathrm{H}-\mathrm{up}$ to H-down unit-cells.

Fig[1](a,b) shows plan views of H-up and Hdown water bilayer models, for which we have constructed He-surface interaction potentials, while panels $(c, d)$ presents iso-surfaces for the attractive well of the H-up (panel c) and Hdown (panel d) potentials. Both iso-surfaces demonstrate that the well is complicated and can not be described with simple continuum surfaces within the unit-cell, hence the effect of the well on the scattering event can not be accounted for by using beeby correction as enhancement to eikonal approximation. ${ }^{14,19}$ Furthermore, the attractive well is markedly different between the two models, which will affect the scattering at low energies. We have considered several proton-disordered surfaces as well. To avoid an unreasonable computing time, the disorder at the upper part of the bilayer (random H-up and H-down) was represented using super-cell of $3 \times 3$ water unit-cells, and different ratios of H-up to H-down. A typical result is illustrated in fig[1](e), for a super-cell with 6:3 $\mathrm{H}$-up to H-down. An iso-surface of $\mathrm{V}=-10 \mathrm{meV}$ has been superimposed on a plan view of the structure. The bottom of the potential well (of which the iso-surface is a measure) is corrugated in a semi-random fashion, governed by the H-up to H-down ratio.

The scattering calculations were performed using the close coupling method, ${ }^{28,29}$ in which the time independent Schrödinger equation is solved. We have used the "Multiscat" code developed originally by Manolopoulos et al. ${ }^{25,30}$ One advantage of using an exact method to simulate the scattering is the ability to observe bound-state resonances ${ }^{31,32}$ (BSR). BSR occur when at certain kinematic conditions the helium atom is scattered so as to travel for some time parallel to the surface before emerging in an allowed diffraction channel. A characteristic of the presence of BSR is multiple scattering and a strong dependence of the diffraction pattern on the beam energy, where the resonances themselves may be peaks, dips or have a Fano like profile. ${ }^{29}$

Fig[2](a) presents the diffraction intensities for H-up model, for the [10], [00] and [10] diffraction channels. For each channel, the diffraction intensity is represented with bars which stands for different beam energy (5$17 \mathrm{meV}$ ). Each bar is normalized to the corresponding bar in the [00] channel. The diffraction pattern is highly dependent on the beam energy. We attribute this strong dependence to scattering through bound-state resonances, as 

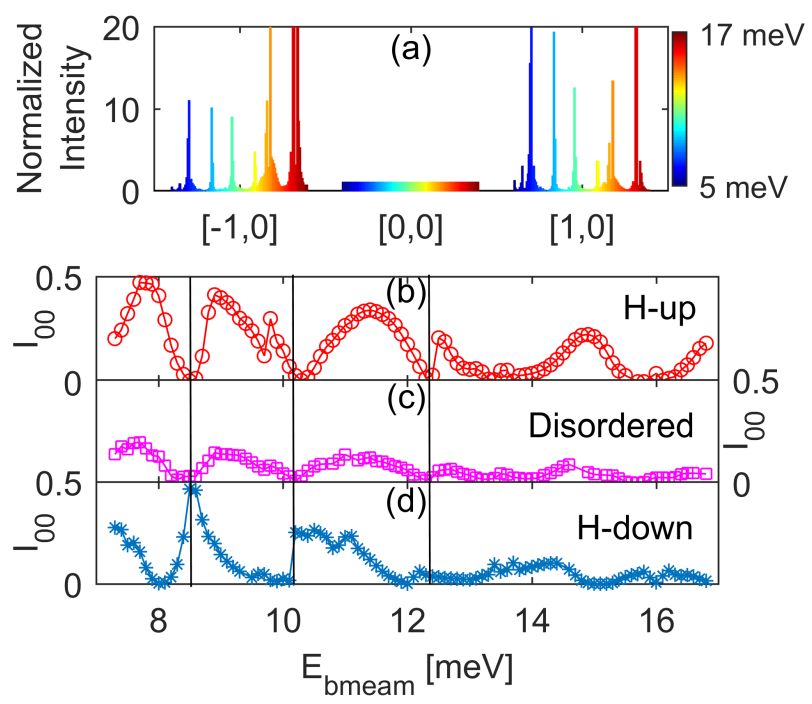

Figure 2: Calculated diffraction intensities from H-up, H-down and pseudo-disordered models. (a) Diffraction for H-up model. Each bar within each channel ([10], [00], [10]) represent different beam energy $(5-17 \mathrm{meV})$ and is normalized to the corresponding $\mathrm{I}_{00}$ bar. (bd) Specular intensity as function of beam energy for H-up, H-down and pseudo-disordered models. The vertical lines emphasize the similarity between bound-state resonances of the pseudo-disordered and H-up models, explained by the $\mathrm{H}$-up/H-down ratio $6 / 3$ of the pseudodisordered model which tends towards H-up.

described above. The significant contribution from bound-state resonances is strong evidence that the eikonal approximation is not appropriate to describe the scattering event, as both multiple scattering and the attractive well play an important role.

Fig[2](b-d) presents the energy dependence of the specular channel for both H-up (top panel) and H-down (bottom panel) models, and for the pseudo-disordered structure (centeral panel) presented on fig $[1(\mathrm{e})]$. As expected, resonances exist at various beam energies, with different resonance energies for the different proton ordering. The disorder can be seen to decrease the amplitude of the resonances as well as broadening the resonant features. Further more, the disordered surface resonances are similar to the $\mathrm{H}$-up resonances, resulted from the ratio of $\mathrm{H}$-up to $\mathrm{H}$-down is $6 / 3$ in this particular pseudo-disordered surface. Calculating a pseudo-disordered surface with the inverse ratio yielded a dependency closer to that of the H-down model. The broadening of the bound-states features for the disordered model implies that the life-time of the bound-states are shorter. ${ }^{29}$ This result shows that the degree of disorder and the ratio of H-up to H-down can be inferred from the bound-state resonances (BSR), and is a strong motivation for experimental work to study Helium-ice BSR.

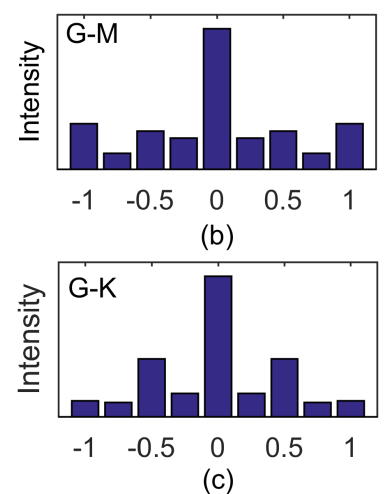

(c)

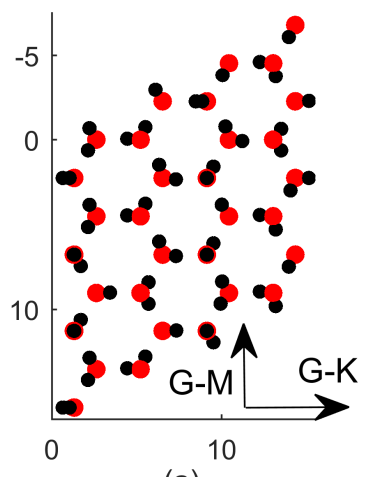

(a)
Figure 3: (a) 4x4 super-cell for Fletcher like model. Red(black) circle represents oxygen(hydrogen) atoms. The main feature of such a model is the H-up rows every second primitive unit-cell. (b-c) Diffraction intensities for 6 fletcher domains which are rotated with $60^{\circ}$ with respect to each other, along $\overline{\Gamma M} \& \overline{\Gamma K}$ azimuth of one of the domains. The bar marked with " 1 " correspond to the $1^{\text {st }}$ order channel of a simple bilayer unit-cell, which is also the $4^{\text {th }}$ order channel with respect to the super-cell. The intensity of half order peak is significant (mainly on $\overline{\Gamma K}$ azimuth).

We now address evidence for the 'Fletcher' model at the surface of ice-Ih. Fig[3(b-c)] present a sum of contributions from the domains of water structured in a Fletcher model fashion (fig[3](a)). The governing property of the Fletcher model is the presence of dangling Hydrogen atoms (H-up) every second unit-cell (see fig[3]), hence it is expected that the half order intensities will be pronounced, as seen in fig[3](c). Previous HAS experiments ${ }^{18,19}$ show strong and narrow $1^{\text {st }}$ order peaks, while the half order peaks are weak, broad and not well 
defined. In contrast, the calculated diffraction pattern for a Fletcher like model shows that the intensity would be almost even between the $1^{\text {st }}$ and half order peaks along the $\overline{\Gamma M}$ direction. We expect that a larger model (than used for our calculation) will preserve this characteristic. For the $\overline{\Gamma K}$ direction, the calculated half order peaks have three times more intensity than the $1^{\text {st }}$ order ones. Our results suggest that the Fletcher model cannot be present at the surface to a significant degree. The previously reported half order peaks ${ }^{19}$ may arise from a co-existance of the Fletcher structure, if it exists, together with other structures with similar energy. ${ }^{8}$

In conclusion, we demonstrate the importance of including multiple-scattering with a realistic potential for computing the scattering of low energy Helium atoms from water surfaces, implying that previous conclusions based on the eikonal method should be reconsidered. The diffraction of helium scattered from water surfaces is highly dependent on the beam energy, with bound state features that are sensitive to the proton ordering. Disorder results a significant decrease in the specular intensity, broaden the bound-state resonance features hence shortening the bound-state resonances life-time. Finally, comparing experimental results from the literature with the diffraction calculations suggest that previously measured ice-surface ${ }^{19}$ is unlikely to be dominated by 'Fletcher' model.

Acknowledgement We are grateful for useful discussions with D.J. Ward, P.S.M Townsend, A. Tamtögl, I. Calvo-Almazán, B.J. Hinch, A.P. Jardine, O. Godsi, G.Corem, G. Alexandrowicz and J. Ellis. For financial support, N. Avidor gratefully acknowledges the Blavatnik Foundation.

\section{References}

(1) Carrasco, J.; Hodgson, A.; Michaelides, A. A Molecular Perspective of Water at Metal Interfaces. Nature Materials 2012, 11, 667-674.

(2) Maier, S.; Salmeron, M. In Surface and Interface Science; Wandelt, K., Ed.; Wiley-
VCH Verlag GmbH \& Co. KGaA, 2016; pp 357-390.

(3) Hodgson, A.; Haq, S. Water Adsorption and the Wetting of Metal Surfaces. Surface Science Reports 2009, 64, 381.

(4) Sun, Z.; Pan, D.; Xu, L.; Wang, E. Role of Proton Ordering in Adsorption Preference of Polar Molecule on Ice Surface. Proceedings of the National Academy of Sciences 2012, 109, 13177-13181.

(5) Kawada, S. Dielectric Dispersion and Phase Transition of $\mathrm{KOH}$ Doped Ice. Journal of the Physical Society of Japan 1972, 32, 1442-1442.

(6) Buch, V.; Groenzin, H.; Li, I.; Shultz, M. J.; Tosatti, E. Proton Order in the Ice Crystal Surface. Proceedings of the National Academy of Sciences 2008, 105, 5969-5974.

(7) Pedersen, A.; Wikfeldt, K. T.; Karssemeijer, L.; Cuppen, H.; Jónsson, H. Molecular Reordering Processes on Ice (0001) Surfaces from Long Timescale Simulations. The Journal of Chemical Physics 2014, $141,234706$.

(8) Pan, D.; Liu, L.-M.; Tribello, G. A.; Slater, B.; Michaelides, A.; Wang, E. Surface Energy and Surface Proton Order of Ice Ih. Physical Review Letters 2008, 101, 155703 .

(9) Pan, D.; Liu, L.-M.; Tribello, G. A.; Slater, B.; Michaelides, A.; Wang, E. Surface Energy and Surface Proton Order of the Ice Ih Basal and Prism Surfaces. Journal of Physics: Condensed Matter 2010, 22, 074209.

(10) Engel, E. A.; Monserrat, B.; Needs, R. J. Vibrational Effects on Surface Energies and Band Gaps in Hexagonal and $\mathrm{Cu}_{-}$ bic Ice. The Journal of Chemical Physics 2016, 145, 044703. 
(11) Bockstedte, M.; Michl, A.; Kolb, M.; Mehlhorn, M.; Morgenstern, K. Incomplete Bilayer Termination of the Ice (0001) Surface. The Journal of Physical Chemistry C 2016, 120, 1097-1109.

(12) Nie, S.; Feibelman, P. J.; Bartelt, N. C.; Thürmer, K. Pentagons and Heptagons in the First Water Layer on Pt(111). Physical Review Letters 2010, 105, 026102.

(13) Smith, J. R.; Ying, S. C.; Kohn, W. Chemisorbed Hydrogen: Electron Scattering, Resonance Levels, and Vibration Frequency. Solid State Communications 1974, 15, 1491-1494.

(14) Farias, D.; Rieder, K. H. Atomic Beam Diffraction from Solid Surfaces. Reports on Progress in Physics 1998, 61, 1575.

(15) Haq, S.; Hodgson, A. Multilayer Growth and Wetting of $\mathrm{Ru}(0001)$. The Journal of Physical Chemistry C 2007, 111, 59465953.

(16) Avidor, N.; Hedgeland, H.; Held, G.; Jardine, A. P.; Allison, W.; Ellis, J.; Kravchuk, T.; Alexandrowicz, G. Highly Proton-Ordered Water Structures on Oxygen Precovered $\mathrm{Ru}\{0001\}$. The Journal of Physical Chemistry A 2011, 115, 72057209 .

(17) Gallagher, M.; Omer, A.; Darling, G. R.; Hodgson, A. Order and Disorder in the Wetting Layer on $\mathrm{Ru}(0001)$. Faraday Discussions 2009, 141, 231-249.

(18) Braun, J.; Glebov, A.; Graham, A. P.; Menzel, A.; Toennies, J. P. Structure and Phonons of the Ice Surface. Physical Review Letters 1998, 80, 2638.

(19) Glebov, A.; Graham, A. P.; Menzel, A.; Toennies, J. P.; Senet, P. A Helium Atom Scattering Study of the Structure and Phonon Dynamics of the Ice Surface. The Journal of Chemical Physics 2000, 112, 11011-11022.
(20) Suter, M. T.; Andersson, P. U.; Pettersson, J. B. C. Surface Properties of Water Ice at $150-191 \mathrm{~K}$ Studied by Elastic Helium Scattering. The Journal of Chemical Physics 2006, 125, 174704.

(21) Wei, X.; Miranda, P. B.; Zhang, C.; Shen, Y. R. Sum-Frequency Spectroscopic Studies of Ice Interfaces. Physical Review B 2002, 66, 085401.

(22) J. Bisson, P.; Jane Shultz, M. Hydrogen Bonding in the Prism Face of Ice Ih via Sum Frequency Vibrational Spectroscopy. The Journal of Physical Chemistry A 2013, 117, 6116-6125.

(23) Rieder, K.-H. In Helium Atom Scattering from Surfaces; Hulpke, D. E., Ed.; Springer Series in Surface Sciences 27; Springer Berlin Heidelberg, 1992; pp 4171.

(24) Manson, J. R. In Helium Atom Scattering from Surfaces; Hulpke, D. E., Ed.; Springer Series in Surface Sciences 27; Springer Berlin Heidelberg, 1992; pp 173205.

(25) Riley, D. J.; Jardine, A. P.; Alexandrowicz, G.; Hedgeland, H.; Ellis, J.; Allison, W. Analysis and Refinement of the $\mathrm{Cu}(001) \mathrm{c}(2 \times 2) \mathrm{CO}-\mathrm{He}$ Potential using He3 Selective Adsorption Resonances. The Journal of Chemical Physics 2008, 128, 154712 .

(26) Choi, B. H.; Graham, A. P.; Tang, K. T.; Toennies, J. P. Helium Atom Scattering from Isolated $\mathrm{CO}$ Molecules on a Pt(111) Surface: Experiment Versus Close-Coupling Calculations for a Realistic He-CO Potential. The Journal of Chemical Physics 2000, 112, 1053810547.

(27) Hodges, M. P.; Wheatley, R. J.; Harvey, A. H. Intermolecular Potential and Second Virial Coefficient of the WaterHelium Complex. The Journal of Chemical Physics 2002, 116, 1397-1405. 
(28) Wolken, G. J. Theoretical Studies of Atom-Solid Elastic Scattering: $\mathrm{He}+\mathrm{LiF}$. The Journal of Chemical Physics 1973, 58, 3047-3064.

(29) Sanz, A. S.; Miret-Artés, S. Selective Adsorption Resonances: Quantum and Stochastic Approaches. Physics Reports 2007, 451, 37-154.

(30) Manolopoulos, D. E.; Wyatt, R. E.; Clary, D. C. Iterative Solution in Quantum Scattering Theory. The Log dDrivative Kohn Approach. Journal of the Chemical Society, Faraday Transactions 1990, 86, 1641-1648.

(31) Kraus, P.; Tamtögl, A.; MayrhoferReinhartshuber, M.; Apolloner, F.; Gösweiner, C.; Miret-Artés, S.; Ernst, W. Surface Structure of Bi(111) from Helium Atom Scattering Measurements. Inelastic Close-Coupling Formalism. The Journal of Physical Chemistry C 2015, 119, 17235-17242.

(32) Lennard-Jones, J.; Devonshire, A. Diffraction and Selective Adsorption of Atoms at Crystal Surfaces. Nature 1936, 137, 1069. 